\title{
A Differential Game Model of Opinion Dynamics: Accord and Discord as Nash Equilibria
}

\author{
Muhammad Umar B. Niazi ${ }^{1,2} \cdot$ A. Bülent Özgüler ${ }^{1}$ \\ Published online: 26 March 2020 \\ (c) Springer Science+Business Media, LLC, part of Springer Nature 2020
}

\begin{abstract}
This paper presents a noncooperative differential (dynamic) game model of opinion dynamics with open-loop information structure. In this game, the agents' motives are shaped by their expectations of the nature of others' opinions as well as how susceptible they are to get influenced by others, how stubborn they are, and how quick they are willing to change their opinions on a set of issues in a prescribed time interval. These motives are independently formed by all agents. The existence of a Nash equilibrium in the network means that a collective behavior emerges out of local interaction rules and these individual motives. We prove that a unique Nash equilibrium may exist in the game under quite different circumstances. It may exist not only if there is a harmony of perceptions among the agents of the network, but also when agents have different views about the correlation among issues. The first leads to an accord in the network usually expressed as a partial consensus, and the second to a discord in the form of oscillating opinions. In the case of an accord, the harmony in the network may be in the form of similarity in pairwise conceptions about the issues but may also be an agreement on the status of a "leader" in the network. A Nash equilibrium may fail to exist only if the network is in a state of discord.
\end{abstract}

Keywords Opinion dynamics · Partial consensus · Dynamic game theory · Optimal control

This work is supported by the Science and Research Council of Turkey (TÜBÍTAK) under the project EEEAG-114E270.

Electronic supplementary material The online version of this article (https://doi.org/10.1007/s13235-02000350-3) contains supplementary material, which is available to authorized users.

Muhammad Umar B. Niazi

niazi@ee.bilkent.edu.tr; muhammad-umar-b.niazi@gipsa-lab.fr

A. Bülent Özgüler

ozguler@ee.bilkent.edu.tr

1 Department of Electrical and Electronics Engineering, Bilkent University, 06800 Ankara, Turkey

2 Present Address: Univ. Grenoble Alpes, CNRS, Inria, Grenoble INP, GIPSA-Lab, 38000 Grenoble, France 


\section{Introduction}

The models describing opinion dynamics aim to explain the situations of conflict and conformity in a society. These models not only find applications in social sciences and economics, but also have a variety of applications in the fields of engineering and computer science $[35,40,41]$. They have thus attracted a diverse community of researchers in quite different fields as evidenced by $[1,26,29,30]$, in which several such models are surveyed.

The early DeGroot's model [12] of weighted averages focused on how consensus is reached in a network of agents. Friedkin-Johnsen (FJ) model [16] incorporated a "stubbornness" component of agents in the DeGroot's model. However, as mentioned in [28], situations of conflict are quite common in a social network, and consensus may not always reach. To capture such situations, the bounded confidence models like those of Hegselmann-Krause (HK) [20] and Deffuant-Weisbuch [11] aim to explain disagreements in the form of network clustering and polarization. Several variations of HK model with convergence rate analysis have been presented in $[9,14,31,32]$. On the other hand, the number of issues on which the agents may form their opinions can be more than one, and the issues can also be interlinked and correlated with each other. In [38], a multidimensional extension of FJ model along with an analysis of stability and convergence is presented. However, the role of dependence and correlation among multiple issues is not highlighted in [38]. Moreover, it must be emphasized that in all these works, the models are deterministic (as opposed to, e.g., [7]) in the form of linear, sometimes time-varying, difference or differential equations. They examine the evolution of the resulting solutions that represent opinion trajectories. Such modeling efforts culminated in [4], which take a state-dependent approach of allowing that an agents' susceptibilities to persuasion are functions of the agents' current beliefs.

Another research track is represented by game-theoretic models of $[8,15,18,19]$. In [8], a game where every node in a network optimizes a quadratic cost function is formulated. The Nash equilibrium of the game is compared with the social optimal solution, which yields a Price of Anarchy in an opinion game. Finite opinion games in [15] examine equilibria (Nash as well as socially optimal) under the assumption that the strength of opinion on an issue is not measured by a continuum but can only have binary values, similar to the voter model of [46]. Independently of $[8,15,19]$, an $\mathrm{N}$-person opinion game with quadratic costs that take into account influence and stubbornness of agents is examined in [18]. A more comprehensive setup in [19] looks at a class of games based on the concept of dissonance minimization and offers a unifying perspective on a number of models, linking them to rational choice theory. All four game-theoretic approaches in $[8,15,18,19]$ consider static games of opinion dynamics on a single issue. Because they are static games, the Nash equilibria are based on the agents' instantaneous responses to opinions. The convergence or stability studies are all relative to the best response dynamics of these static games.

In a static game of opinion dynamics, each agent chooses its opinion to minimize its individual cost function by taking into account the presently observed opinions of its neighbors. However, in the games where agents also influence and manipulate others, not only each agent examines its neighbors' opinions, but also observes their strategies to choose those opinions. Agents may judge the opinions of others based on the environmental priors like social structure, culture, ethnicity, and religion. At least in the formation of political opinions, this has been empirically supported by studies [24]. Unlike static games, the dynamic approach presented in this paper allows agents to project their beliefs and expectations of their neighbors' behavior into an integral cost function. In a multiple issue game, they can further reflect their biases on the correlations among the issues into the cost function. Integral 
cost functions are also employed in [2] with a quite different objective of external control of consensus via suitable inputs. The dynamic game on multiple issues studied here is inspired by the foraging swarm models in $[37,44,45]$.

We present a noncooperative differential (dynamic) game model of opinion dynamics in a society, where the agents' motives are shaped by how susceptible they are to get influenced by others, how stubborn they are, and how quick they are willing to change their opinions on a set of issues in a prescribed time interval. We thus consider a multiple issue dynamic game. We allow the agents to project their beliefs and expectations of their neighbors' behavior into the cost functions, as well as how much they are willing to revise their initial opinions. The cost functions describe the motives of the agents, which are the social and psychological disposition of agents - a compromise between their personal biases and desire to conform with their neighbors in the network.

In bounded confidence models, for instance, agents' susceptibilities to influence depend on their initial and current beliefs. In choosing the weight parameters and incorporating them into the cost functions, agents may thus take bounded confidence into account. Each agent is assumed to independently control the rate of change of its opinion on each issue. The main objective of our investigation is to determine under what conditions on the motives and the network structure a unique Nash equilibrium exists.

There are of course many notions of equilibrium in games along with Nash equilibrium $[6,13,36]$. An interpretation in [36] for static games suggests that if a game is played several times without any strategic links between consecutive plays, then a Nash equilibrium is most likely reached. Nash equilibrium is a useful construct whenever the objective is to investigate under what conditions a pattern of collective behavior emerges from independent motives of agents. It is important to note that no agent in this game is assumed to ever explicitly calculate or know its best response function. They would rather respond and behave somewhat like that because they put (mostly unconscious) efforts toward optimizing their cost functions.

The features that put the model here apart from those in the literature are the following: (1) It is a game-theoretic model played by the agents in a society but a dynamic game as opposed to static; not instantaneous but accumulated costs are minimized. This allows the agents to reflect their expectations in a time interval into their objective functionals. (2) It is a noncooperative game, and our focus is on a unique Nash equilibrium that has the property that if an agent uses its best response strategy, then he/she is not worse off even when all the others play their best strategies. (3) It is a multiple issue game, the most prominent difference of which comes from being able to incorporate an expectation on the nature of correlation between any pair of issues into the cost functional.

The main conclusion reached by our study is that a unique Nash equilibrium results under certain conditions on the information structure, and it is often a state of accord in the network. This collective behavior is a partial consensus. A unique Nash equilibrium may also be a state of discord (in which the opinion trajectories oscillate throughout the time interval) in certain other information structures. Such structures may also cause a Nash equilibrium (unique or non-unique) fail to exist.

Opinion profiles of discord at Nash equilibrium are interesting and deserve a closer look. They are essentially caused by conflicts of conceptions of agents on correlation among the prevailing issues. The agents are captured between two pressures to update their opinions on the issues. On the one hand, their conceptions of correlation between issues $\mathrm{X}$ and $\mathrm{Y}$ are diametrically opposite, while, on the other hand, they are susceptible to be influenced positively by their neighbors. Let us suppose, as consistency necessitates, that the agents maintain their conception of correlation between issues $\mathrm{X}$ and $\mathrm{Y}$ in their opinions. If two agents are at a perfect agreement at some time instant on issue $\mathrm{X}$ but have a disagreement on 
issue Y, no matter how small, then an oscillatory opinion profile may result as follows. Since it believes that issues $\mathrm{X}$ and $\mathrm{Y}$ are, say, positively correlated, one agent will tend to change his opinion to differ with the second agent on issue $\mathrm{X}$, and his difference of opinion on $\mathrm{X}$ will get into an upward climb. The other agent, believing that the two issues are negatively correlated, updates her opinion in the opposite direction and starts a decline to increase the difference still more. This goes on until the difference between their opinions gets very large that the pressure to be positively influenced by each other compels both agents to reduce their difference of opinions, causing an approach until their opinions on issue $\mathrm{X}$ again coincide. This periodically continues to result in a sinusoidal opinion profile on issue X. By the inherent symmetry in the correlation conception, a similarly sinusoidal profile also results on issue Y.

Dynamics of an opinion, even on a single issue, may exhibit oscillations in some situations as illustrated by the evolution of certain Wikipedia articles on controversial issues, [25]. The state of discord in our model pertains to multiple issues and is caused by differing assumptions of agents on the nature of correlation between issues. The differences of opinion on a single issue may also grow indefinitely as predicted by certain nonlinear models. The model [17] gives an example of a dyad (two agents) that results in an unstable deadlock dynamics, in which the opinions of the agents end up in a discord of arbitrarily large size given a sufficiently large duration of debate. The growing oscillations that result in a Nash equilibrium failing to exist, in our case, are again a distinctive feature of the multiple issue game.

The paper is organized as follows. In Sect. 2, we define a dynamic game of opinion dynamics on multiple issues. In Sect. 3, we determine in Theorem 1 the exact conditions for existence of a unique Nash equilibrium and describe the resulting opinion trajectories of agents. In Theorem 2, the conditions under which a Nash equilibrium results in an accord or discord are determined. By making suitable assumptions on the network topology, we then study, in Corollary 1 and 2, two somewhat extreme situations in which an accord in the form of (partial) consensus results. An application example involving a large number of agents is presented in Sect. 4, where three typical Nash profiles, one being oscillatory along the lines outlined in the previous paragraph, are illustrated. Following Sect. 5 on Concluding Remarks, the "Appendix" contains the more technical parts of the proofs of Theorems 1 and 2 together with a characterization of non-unique Nash equilibria that may also exist. We abide by the following notations throughout the paper:

Notation: Vectors are denoted by bold lowercase letters, e.g., $\mathbf{x}$, and matrices by uppercase letters, e.g., $X$. The set of real numbers and nonnegative real numbers are denoted by $\mathbb{R}$ and $\mathbb{R}_{\geq 0}$, respectively. The Euclidean norm of a vector $\mathbf{x} \in \mathbb{R}^{n}$ is denoted by $|\mathbf{x}|$. For sets $A$ and $B, A \backslash B$ denotes those elements of set $A$ that are not in set $B$. For any set $A,|A|$ denotes its cardinality. The $n \times 1$ vector of all ones is $\mathbf{1}_{n}$ and $n \times n$ identity matrix is $I_{n}$, where the subscript is sometimes omitted for brevity. The transpose of a matrix $A$ is denoted by $A^{\prime}$. The $i j$ th entry of a matrix $W$ is denoted by $w_{i j}$ or $w^{i j}$ (lowercase letter) so that $W_{i j}$ is reserved for a matrix in some matrix array. The operator $\otimes$ denotes the Kronecker product and $\mathscr{L}$ the Laplace transform. A (block) diagonal matrix with matrices $A, \cdots, Z$ at its diagonal is written as $\operatorname{diag}[A, \cdots, Z]$.

\section{A Game of Multidimensional Opinion Dynamics}

Consider a network where $n$ agents indexed by a set $\mathrm{N}=\{1, \cdots, n\}$ interact, discuss, and form opinions on $d$ prevailing issues in a set D. Every agent $i$ has some initial opinions 
$\mathbf{b}_{i}=\left[b_{i}^{1} \cdots b_{i}^{d}\right]^{\prime} \in \mathbb{R}^{d}$ on the issues. Here, we let $b_{i}^{k}$ assume any number in $\mathbb{R}=(-\infty, \infty)$ ranging from strong refusal to complete support, with value zero indicating neutrality or lack of opinion. People may update their initial opinions in a time interval $[0, \tau]$, where $\tau>0$ may also tend to infinity. Let $\mathbf{x}_{i}(t)=\left[x_{i}^{1}(t) \cdots x_{i}^{d}(t)\right]^{\prime} \in \mathbb{R}^{d}$ denote the vector of opinions of agent $i$ at time $t \in[0, \tau]$ on $d$ issues. Thus, $\mathbf{x}_{i}(0)=\mathbf{b}_{i}$. The motive that compels agent $i$ to update its opinions can be described by a cost functional that takes into account the cumulative costs of (i) rapid changes in one's opinions, (ii) holding different opinions from one's neighbors, and (iii) changing one's initial opinions (mind set). The model assumes that every agent has a motive that may eventually dictate its opinion dynamics and that such a motive, if not consciously held, looms at the background in shaping its opinions. The cost functional of agent $i$ given by

$$
\mathscr{J}_{i}\left(\mathbf{x}, \mathbf{b}_{i}, \mathbf{u}_{i}\right)=\frac{1}{2} \int_{0}^{\tau}\left\{\left[\sum_{j \in \mathrm{N}}\left(\mathbf{x}_{i}-\mathbf{x}_{j}\right)^{\prime} W_{i j}\left(\mathbf{x}_{i}-\mathbf{x}_{j}\right)\right]+\left(\mathbf{x}_{i}-\mathbf{b}_{i}\right)^{\prime} W_{i i}\left(\mathbf{x}_{i}-\mathbf{b}_{i}\right)+\mathbf{u}_{i}^{\prime} \mathbf{u}_{i}\right\} d t
$$

represents its motive, in which the matrices $W_{i j}, W_{i i} \in \mathbb{R}^{d \times d}$ weigh the costs in (ii) and (iii) above, respectively. The third term penalizes (i) as, $\forall i \in \mathrm{N}, \mathbf{u}_{i}(t):=\dot{\mathbf{x}}_{i}(t)$, i.e., it is the instantaneous rate of change of opinions of agent $i$ on the issues $\mathrm{D}$ and represents the cumulative control effort of agent $i$ when integrated in (1). The functions $\mathbf{x}$ and $\mathbf{u}$ depend on time $t$ and the terminal time $\tau$. Throughout the paper, however, we drop $\tau$, and sometimes also $t$, for the sake of brevity. These independent variables should be considered implicit in the notations $\mathbf{x}$ and $\mathbf{u}$. We adopt the following throughout the paper.

Assumption $1 W_{i i}$ is symmetric positive definite and $W_{i j}, i \neq j$, is symmetric, i.e., $W_{i j}=W_{i j}^{\prime}$, and positive semi-definite.

The first term in the cost function is formed by agent $i$ based on its perception and expectation of how the opinion of agent $j$ will evolve. It is obviously based on a good deal of judgment. Whether agent $i$ predicts the behavior of agent $j$ in the interval $[0, \tau]$ close enough or not has a great bearing on the existence of a solution to the game. If agent $i$ has no reliable information to speculate on how agent $j$ may form and revise an opinion, it can choose the corresponding entry in the weight $W_{i j}$ to be zero. A choice $W_{i j}=0$ is even allowed and would mean that $i$ does not consider $j$ as a "neighbor." The first term thus reflects, via the choice of the weight, a motive of agent $i$ to get influenced by agent $j$. The second term is to preserve its own biases, and the third term to reduce the overall control effort. By Assumption 1, the weight associated with the control effort, normalized to identity in (1) rather than allowing it to be a positive definite weight matrix, is without loss of generality.

The agents interact with their neighbors for a finite interval $[0, \tau]$ and, due to an integral cost, every agent penalizes the cumulative effect of each of the three terms in the integrand during that interval. For instance, the first term does not penalize the instantaneous differences but the sum total of divergence from the opinions of the neighbors. This is one of the main distinctive features of the model (1). The opinions $\mathbf{x}_{i}(\tau)$ at terminal time $\tau$ are not specified and left free. Therefore, each agent minimizes its cost under free terminal conditions. A noncooperative, continuous-time, open-loop, infinite dynamic game (see [6], Section 5.3) is thus played by $n$ agents: For every $i \in \mathrm{N}$,

$$
\begin{array}{cl}
\min _{\mathbf{u}_{i}} & \mathscr{J}_{i}\left(\mathbf{x}, \mathbf{b}_{i}, \mathbf{u}_{i}\right) \\
\text { subject to } & \left\{\begin{array}{l}
\dot{\mathbf{x}}_{i}(t)=\mathbf{u}_{i}(t) \\
\mathbf{x}_{i}(0)=\mathbf{b}_{i}
\end{array} .\right.
\end{array}
$$


The vector $\mathbf{x}(t)=\left[\mathbf{x}_{1}^{\prime}(t) \cdots \mathbf{x}_{n}^{\prime}(t)\right]^{\prime}$ is the opinion profile of the agents at $t \in[0, \tau]$. This game is noncooperative because the agents seek their own individual motives, and there is no prevailing "social motive" in the network. We assume that, $\forall t \in[0, \tau], \mathbf{u}_{i}(t) \in \mathrm{S}_{i}$ and define $S_{i}$ to be a class of all permissible strategies of agent $i$, which are all continuously differentiable functions $\mathbf{s}_{i}:[0, \tau] \times \mathbb{R}^{n d} \rightarrow \mathbb{R}^{d}$. A set of permissible strategies $\left(\mathbf{u}_{1}^{*}, \cdots, \mathbf{u}_{n}^{*}\right)$, where $\mathbf{u}_{i}^{*}(t):=\mathbf{s}_{i}^{*}(t, \mathbf{b})$, constitute a Nash equilibrium of the game (2) if, for all strategies $\mathbf{u}_{i}(t)=\mathbf{s}_{i}(t, \mathbf{b})$, it holds that

$$
J_{i}\left(\mathbf{u}_{1}^{*}, \cdots, \mathbf{u}_{i-1}^{*}, \mathbf{u}_{i}^{*}, \mathbf{u}_{i+1}^{*}, \cdots, \mathbf{u}_{n}^{*}\right) \leq J_{i}\left(\mathbf{u}_{1}^{*}, \cdots, \mathbf{u}_{i-1}^{*}, \mathbf{u}_{i}, \mathbf{u}_{i+1}^{*}, \cdots, \mathbf{u}_{n}^{*}\right)
$$

for all $\mathbf{b}=\left[\mathbf{b}_{1}^{\prime} \cdots \mathbf{b}_{n}^{\prime}\right]^{\prime} \in \mathbb{R}^{n d}$, where $J_{i}\left(\mathbf{u}_{1}, \cdots, \mathbf{u}_{n}\right)$ denotes the value of (1) resulting from the strategy $\mathbf{u}_{i}=\mathbf{s}_{i}(t, \mathbf{b})$, for all $i \in \mathrm{N}$. An opinion profile that results from a Nash equilibrium $\left(\mathbf{u}_{1}^{*}, \cdots, \mathbf{u}_{n}^{*}\right)$ will be denoted by $\mathbf{x}^{*}=\left[\left(\mathbf{x}_{1}^{*}\right)^{\prime} \cdots\left(\mathbf{x}_{n}^{*}\right)^{\prime}\right]^{\prime}$ and referred to as a Nash profile.

We define the neighborhood of agent $i, \mathrm{~N}_{i} \subset \mathrm{N} \backslash\{i\}$, by $j \in \mathrm{N}_{i}$ if $W_{i j} \neq 0$. Thus, any agent with whom agent $i$ expects to have no contact in $[0, \tau]$ is left out and others are collected in $\mathrm{N}_{i}$ as neighbors. The summation in (1) can thus be taken over $\mathrm{N}_{i}$ in place of $\mathrm{N}$. We can think of the society envisaged here as a network, in which every agent $i$ is a vertex connected to its neighbor $j \in \mathrm{N}_{i}$ by an edge that has weights $w_{i j}^{k l}$ (the $k l$ th entry of $W_{i j}$ ) associated with issues $k, l \in \mathrm{D}$. This is then a multiplex directed network of $d$ layers [10].

The entries of $W_{i j} \in \mathbb{R}^{d \times d}$ indicate the $i$-centered view of the issues with respect to its neighbors $j \in \mathrm{N}_{i}$. For instance, for $k=1, \cdots, d$, the diagonal entries $w_{i j}^{k k}$ of $W_{i j}$ indicate whether $i$ considers to agree $w_{i j}^{k k}>0$, disagree $w_{i j}^{k k}<0$, or stay neutral $w_{i j}^{k k}=0$ with $j$ on issue $k$. In light of Assumption 1, we consider $w_{i j}^{k k} \geq 0$, i.e, agent $i$ either agrees or stays neutral with $j$, and that $w_{i j}^{k l}=w_{i j}^{l k}$, for $l \neq k$, i.e., symmetry of views. The off-diagonal entries $w_{i j}^{k l}=w_{i j}^{l k}$ reflect $i$ 's view on the correlation between issues $k$ and $l$ in relation to $j$. That is, when $i$ interacts with $j, w_{i j}^{k l}$ determines the quality and quantity of correlation between issues $k$ and $l$. Note that as $W_{i j}$ is $i$-centered view with respect to $j, W_{j i}$ is $j$-centered view with respect to $i$, which can be different.

If the matrices $W_{i j}$ and $W_{i i}$ are diagonal, then (1) will be minimum if and only if the $d$ functionals obtained by its decomposition are minimum. In other words, the game then decouples into $d$ independently played games on every issue separately, which can be covered by the single issue $(d=1)$ results of [34]. Such a game will always yield partial consensus as Nash equilibrium. A game can yield discord as Nash equilibrium only if agents consider multiple issues that are correlated, i.e., $W_{i j}$ and $W_{i i}$ are non-diagonal matrices. Then, an agent may make consensus with only those neighbors whom it considers to be consistent, according to its own conception of correlation, and, respectively, it may deviate from the opinions of those who hold inconsistent opinions. More details on the choice of weight matrices is provided in Sect. 4.1.

\subsection{Matrix Square Root and Hyperbolic Functions}

In closing this section, we recall certain useful facts concerning the square roots of matrices and some hyperbolic-like matrix functions.

Given a real matrix $Q \in \mathbb{R}^{m \times m}$, a (complex) square root of $Q$ is $H \in \mathbb{C}^{m \times m}$ satisfying $Q=H^{2}$. The conditions for the existence of a square root are given in [22], Chapter 6. A square root always exists for a nonsingular $Q$, and a real square root of a nonsingular $Q$ exists if and only if $Q$ has an even number of Jordan blocks of each size for every negative 
eigenvalue. It is also well known that a real, positive (respectively, positive semi-) definite matrix has a unique real, positive (respectively, positive semi-) definite square root (see [23], Theorem 7.2.6), which will be denoted by $Q^{\frac{1}{2}}$.

Consider the Jordan form $J=P Q P^{-1}$ of a nonsingular $Q$ and note that a square root $H$ of $Q$ can be written as $H=H_{+}+H_{-}$, where $H_{+}=P^{-1} J_{+} P, H_{-}=P^{-1} J_{-} P, J_{+}$and $J_{-}$are matrices corresponding to the jordan blocks with eigenvalues of positive and negative real parts, respectively, and some zero blocks so that $J_{+} J_{-}=J_{-} J_{+}=0$ and $J_{+}^{2}+J_{-}^{2}=J$. We define $H_{p}:=H_{+}-H_{-}$for use in Theorem 2 and note that it is a square root of $Q$ having all its eigenvalues with positive real parts.

Consider the following matrix functions as infinite series:

$$
f_{Q}(t):=\sum_{k=0}^{\infty} Q^{k} \frac{t^{2 k}}{(2 k) !}, \quad g_{Q}(t):=\sum_{k=0}^{\infty} Q^{k} \frac{t^{2 k+1}}{(2 k+1) !}, \quad h_{Q}(t):=\sum_{k=0}^{\infty} Q^{k} \frac{t^{2 k+2}}{(2 k+2) !} .
$$

It is easy to verify that these series, in particular, satisfy the following:

$$
\begin{aligned}
& \frac{d}{d t} g_{Q}(t)=f_{Q}(t), f_{Q}(t-\tau)=f_{Q}(t) f_{Q}(\tau)-Q g_{Q}(t) g_{Q}(\tau), \\
& \frac{d}{d t} h_{Q}(t)=g_{Q}(t), g_{Q}(t-\tau)=g_{Q}(t) f_{Q}(\tau)-f_{Q}(t) g_{Q}(\tau) .
\end{aligned}
$$

Moreover, if $H$ is any square root of $Q$, then, in (3), $f_{Q}(t)=\cosh (H t), H g_{Q}(t)=\sinh (H t)$, and $f_{Q}(t)=I+Q h_{Q}(t)$, which indicate that $\cosh (H t)$ and $H \sinh (H t)$ are independent of the choice of the square root matrix $H$, whereas $\sinh (H t)$ and $\exp ( \pm H t)=\cosh (H t) \pm$ $\sinh (H t)$ are dependent on its choice.

\section{Main Results}

We present the existence and uniqueness results for a Nash equilibrium of the game of opinion dynamics (2). Some derivations and proofs are deferred to the "Appendix".

We first focus on the Nash equilibrium of the game in its most generality. Given (1), let $W=\operatorname{diag}\left[W_{11}, \cdots, W_{n n}\right]$ and

$$
Q=\left[\begin{array}{cccc}
M_{1} & -W_{12} & \cdots & -W_{1 n} \\
-W_{21} & M_{2} & \cdots & -W_{2 n} \\
\vdots & \vdots & \ddots & \vdots \\
-W_{n 1} & -W_{n 2} & \cdots & M_{n}
\end{array}\right]
$$

with

$$
M_{i}=W_{i i}+\sum_{j \in \mathrm{N}_{i}} W_{i j}, \quad \forall i \in \mathrm{N} .
$$

Note that $W_{i j}=0$ in (5) whenever $j \notin \mathrm{N}_{i}$. Also, by Assumption 1, the block diagonal $W$ is positive definite. Whether $Q$ is symmetric, nonsingular, positive semi-definite, or positive definite, etc., depends on the choice of weight matrices as well as the network structure.

Theorem 1 Let Assumption 1 hold. Then,

(i) A unique Nash equilibrium $\left(\mathbf{u}_{1}^{*}, \cdots, \mathbf{u}_{n}^{*}\right)$ of the game (2) exists in the interval $[0, \tau]$ if and only if one of the following equivalent conditions hold: 
(C1) The matrix $f_{Q}(\tau)$ is nonsingular.

(C2) Either $Q$ has no real negative eigenvalue or, whenever it has such an eigenvalue $-r^{2}, r>0, \tau$ is not an odd multiple of $\frac{\pi}{2 r}$.

(ii) When it exists, the Nash equilibrium, in $t \in[0, \tau]$, has the expression

$$
\mathbf{u}^{*}(t)=\left[g_{Q}(t) f_{Q}(\tau)-f_{Q}(t) g_{Q}(\tau)\right] f_{Q}(\tau)^{-1}(Q-W) \mathbf{b},
$$

where $\mathbf{u}^{*}(t)=\left[\mathbf{u}_{1}^{*}(t)^{\prime} \cdots \mathbf{u}_{n}^{*}(t)^{\prime}\right]^{\prime}$, resulting in the Nash profile

$$
\mathbf{x}^{*}(t)=\mathbf{b}+\left[h_{Q}(t) f_{Q}(\tau)-g_{Q}(t) g_{Q}(\tau)\right] f_{Q}(\tau)^{-1}(Q-W) \mathbf{b} .
$$

(iii) A unique Nash profile is constant, i.e., $\mathbf{x}^{*}(t)=\mathbf{b}$, if and only if $\mathbf{b}$ is in the null space of $Q-W$.

Proof Here, we establish the equivalence of (C1) and (C2) and prove (iii). In the "Appendix", we show that $(\mathrm{C} 1)$ is necessary and sufficient for the existence of a unique Nash equilibrium and derive the expressions in (ii).

Let $J_{Q}=P^{-1} Q P$ be the Jordan normal form of $Q$ for a nonsingular, possibly complex, matrix $P$. If $-r^{2}$ is an eigenvalue of $Q$, then a Jordan block $J_{r}$ of any size associated with this negative eigenvalue can be written as $-K_{r}^{2}$, where $K_{r}$ has $r$ at its diagonal, $-(2 r)^{-1}$ at its upper diagonal when it has size two or more, and zeros elsewhere. The corresponding Jordan block of $f_{Q}(\tau)$ is then

$$
f_{J_{r}}(\tau)=\sum_{i \geq 0}(-1)^{i} K_{r}^{2 i} \frac{\tau^{2 i}}{(2 i) !}=\cos \left(K_{r} \tau\right),
$$

the diagonal entries of which are zero if and only if $r \tau$ is an odd multiple of $\pi / 2$. If $Q$ is singular, then let $J_{0}$ be a Jordan block associated with the eigenvalue zero, and note by its series expression that $f_{J_{0}}(\tau)$ has ones at its diagonal, i.e., $f_{J_{0}}(\tau)$ is nonsingular. Similarly, any other eigenvalue $\lambda$ of $Q$ has a positive real part so that a Jordan block $J_{\lambda}$ has a nonsingular square root $H_{\lambda}$ and $f_{J_{\lambda}}(\tau)=\cosh \left(H_{\lambda} \tau\right)$ has eigenvalues all nonzero, i.e., $f_{J_{\lambda}}(\tau)$ is nonsingular.

To prove (iii), note that any $\mathbf{b}$ in the null space of $Q-W$ gives $\mathbf{x}^{*}(t)=\mathbf{b}$ by (7). Conversely, if $\mathbf{x}^{*}(t)=\mathbf{b}$, then, again by (7),

$$
\left[h_{Q}(t) f_{Q}(\tau)-g_{Q}(t) g_{Q}(\tau)\right] f_{Q}(\tau)^{-1}(Q-W) \mathbf{b}=\mathbf{0} .
$$

Taking the derivative with respect to $t$ and using the identities (4), we obtain that $g_{Q}(t-$ $\tau)(Q-W) \mathbf{b}=\mathbf{0}$. Taking the derivative once more, we obtain $f_{Q}(t-\tau)(Q-W) \mathbf{b}=\mathbf{0}$. But, by $(\mathrm{C} 2), f_{Q}(t-\tau)$ is nonsingular for almost all $t$. Therefore, $\mathbf{b}$ must be in the null space of $Q-W$.

Remark 1 (a) A sufficient condition for the existence of a unique Nash equilibrium is that $Q$ has no negative eigenvalues. This condition is always met in the single issue case, of $d=1$, as it can be shown [33] that $Q$ is then positive stable [23].

(b) If $\mathbf{x}_{i}(0)=\mathbf{b}_{i}$ are such that $\mathbf{b}_{i}=\mathbf{b}_{j}$ for all $i, j \in \mathrm{N}$, then $\mathbf{b}$ is called a full initial consensus. A full initial consensus $\mathbf{b}$ is clearly in the null space of $Q-W$ and, by Theorem 1(iii), gives $\mathbf{x}_{i}^{*}(t)=\mathbf{b}_{i}=\mathbf{b}_{j}$ for all $t \in[0, \tau]$ and for all $i, j \in \mathrm{N}$. Among the possible constant Nash profiles, i.e., b such that $(Q-W) \mathbf{b}=0$, the full initial consensus stands out as a structural constant equilibrium since it is a Nash profile independent of neighborhood relations or of the values assigned to $W_{i j}$ matrices. The structure of $Q-W$ implies that all constant profiles $\mathbf{b}$ have the property: the pairwise differences $\mathbf{d}_{i j}=\mathbf{b}_{i}-\mathbf{b}_{j}$ satisfy 
$\sum_{j \in \mathrm{N}_{i}} W_{i j} \mathbf{d}_{i j}=0$, for $i=1, \cdots, n$. This shows that in order to have a constant profile, all pairwise differences must somewhat be "unobservable" through the influence terms of the cost functionals.

(c) Any initial opinion $\mathbf{b} \in \mathbb{R}^{n}$, by orthogonal decomposition theorem, can be uniquely written as $\mathbf{b}=\tilde{\mathbf{b}}+\hat{\mathbf{b}}$, where $\tilde{\mathbf{b}}$ is in the null space of $Q-W$ and $\hat{\mathbf{b}}=\left[\hat{\mathbf{b}}_{1}^{\prime} \cdots \hat{\mathbf{b}}_{n}^{\prime}\right]^{\prime}$ is in the range space of $(Q-W)^{\prime}$. Let $\hat{\mathbf{b}}=(Q-W)^{\prime} \hat{\mathbf{a}}$ for some $\hat{\mathbf{a}} \in \mathbb{R}^{n}$. Using the fact that $W_{i j}=W_{i j}^{\prime}$ and noting that $\hat{\mathbf{b}}_{k}=\sum_{j=1}^{n}\left(W_{k j} \hat{\mathbf{a}}_{k}-W_{j k} \hat{\mathbf{a}}_{j}\right)$ for $k=1, \cdots, n$, it follows that $\sum_{k=1}^{n} \hat{\mathbf{b}}_{k}=0$, i.e., whenever an initial opinion has no component in the null space of $Q-W$, then the average initial opinion of all the agents is neutral on all issues. Also observe by (7) that this decomposition results in a decomposition of the opinion profile into two parts, triggered separately by $\tilde{\mathbf{b}}$ and $\hat{\mathbf{b}}$.

(d) In a similar manner to the proof of equivalence of (C1) and (C2), it can be shown that $g_{Q}(\tau)$ is singular at some $\tau>0$ if and only if $Q$ has a negative eigenvalue $-r^{2}$ and $r \tau=k \pi$ for an integer $k>0$. This fact will be used in completing the proof of Theorem 1 in the "Appendix".

Theorem 2 Let Assumption 1 hold and suppose a unique Nash equilibrium exists. Further, let $Q$ be nonsingular. Then,

(i) The Nash profile has the expression

$$
\mathbf{x}^{*}(t)=\left\{Q^{-1} W+\cosh [H(\tau-t)] \cosh (H \tau)^{-1}\left(I-Q^{-1} W\right)\right\} \mathbf{b}
$$

for any square root $H$ of $Q$.

(ii) If $Q$ has a real negative eigenvalue $-r^{2}$, then for some initial opinions, there are entries of $\mathbf{x}^{*}(t)$ that have sinusoidal oscillations of period $\frac{2 \pi}{r}$, for $\tau \neq \frac{(2 k+1) \pi}{2 r}$, and

$$
\lim _{\tau \rightarrow \frac{(2 k+1) \pi}{2 r}} \sup _{t \in[0, \tau]}\left|\mathbf{x}^{*}(t)\right| \rightarrow \infty
$$

for every nonnegative integer $k$.

(iii) If $Q$ has no real negative eigenvalue, then the Nash profile, as $\tau \rightarrow \infty$, is given by

$$
\mathbf{x}^{i n f}(t)=\left[Q^{-1} W+\exp \left(-H_{p} t\right)\left(I-Q^{-1} W\right)\right] \mathbf{b},
$$

where $H_{p}$ is the square root of $Q$ that has all its eigenvalues with positive real parts defined in Sect. 2.1.

Proof If $Q$ is nonsingular, then a square root $H$ of $Q$ exists, which implies that $f_{Q}(t)=Q h_{Q}(t)+I=\cosh (H t)$ and $H g_{Q}(t)=\sinh (H t)$. Hence, (7) results in (8), proving Theorem 2(i). To prove Theorem 2(ii), let $H$ be any square root of $Q$ and let $J$ be its Jordan normal form so that $H=P J P^{-1}$ for $P$ in the proof of Theorem 1. If $Q$ has a negative eigenvalue $-r^{2}$, then there are Jordan block(s) $J_{r}=\sqrt{-1} K_{r}$ associated with that eigenvalue for which $\cosh \left(J_{r} t\right)=\cos \left(K_{r} t\right)$ for $t \geq 0$. The corresponding block in

$$
P^{-1} \cosh [H(\tau-t)] \cosh (H \tau)^{-1} P=\cosh [J(\tau-t)] \cosh (J \tau)^{-1}
$$

has the expression

$$
\cos \left[K_{r}(\tau-t)\right] \cos \left(K_{r} \tau\right)^{-1}=\cos \left(K_{r} t\right)+\sin \left(K_{r} t\right) \tan \left(K_{r} \tau\right)
$$

It follows that, due to the term $\sin \left(K_{r} t\right)$, some initial values $\mathbf{b}$ (except those in the null space of $Q-W)$, will excite entries of $\mathbf{x}^{*}(t)$ that are oscillatory for every $\tau \in \mathbb{R}$. The amplitude of the oscillations increases as $\tau$ approaches $\frac{(2 k+1) \pi}{2 r}$, for $k=1,2, \cdots$, due to the term $\tan \left(K_{r} \tau\right)$. 

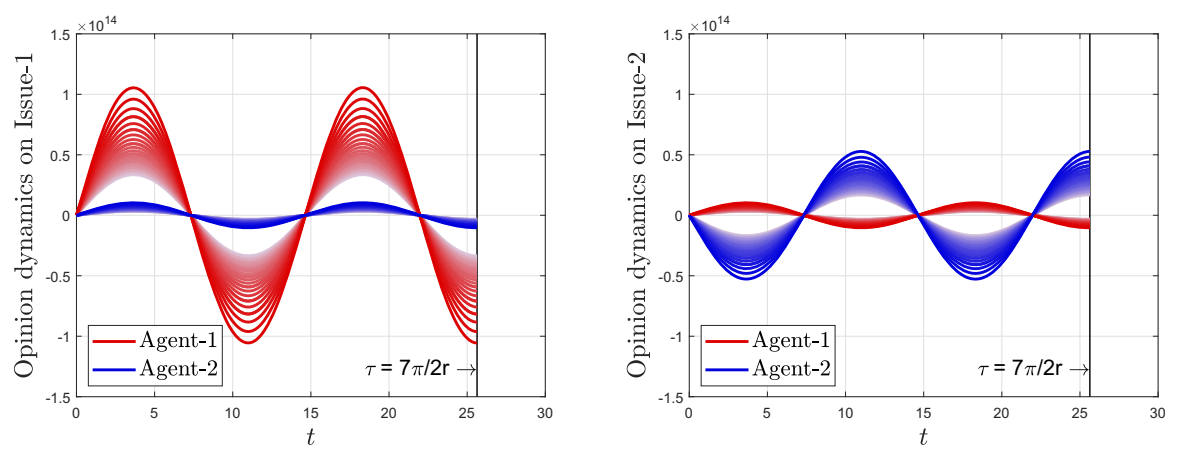

Fig. 1 Oscillating opinion trajectories for $\tau \in\left[c_{3}-10^{-9}, c_{3}\right)$ with increasing magnitude as $\tau \rightarrow c_{3}=7 \pi / 2 r$

The period of the oscillations is determined by the nonzero entries of $\cos \left[K_{r}(\tau-t)\right]$. The matrix $-K_{r}$ has eigenvalue $-r$ so that the period of oscillations is $\frac{2 \pi}{r}$. This proves the claims in Theorem 2(ii). The proof of (iii) is given in the "Appendix".

Example 1 This example illustrates Theorem 2(ii). Consider a dyad, a network of two agents, with

$$
\begin{aligned}
& W_{11}=\left[\begin{array}{cc}
0.5 & -1 \\
-1 & 2.5
\end{array}\right], \quad W_{12}=\left[\begin{array}{cc}
1.25 & -1 \\
-1 & 1.25 \\
2 & 2.5 \\
2.5 & 3.25
\end{array}\right], \\
& W_{22}=W_{21}=\left[\begin{array}{cc}
2 & 1.5 \\
1.5 & 1.25
\end{array}\right]
\end{aligned}
$$

for which $Q$ has an eigenvalue $-0.184 \ldots=-r^{2}$. Then, $c_{k}=\frac{(2 k+1) \pi}{2 r}$, for $k=0,1,2, \ldots$, are the critical values of $\tau$ at which Nash equilibrium fails to exist. For instance, with $\mathbf{b}_{1}=$ $\left[\begin{array}{ll}-0.5 & 0.5\end{array}\right]^{\prime}, \mathbf{b}_{2}=\left[\begin{array}{ll}1 & 1\end{array}\right]^{\prime}$, the opinion profiles have oscillations as shown in Fig. 1, where $\tau \in\left[c_{3}-10^{-9}, c_{3}\right)$ with $c_{3}=7 \pi / 2 r \approx 25$.63. As $\tau \rightarrow c_{3}$, for instance, the oscillations grow in amplitude. Notice that the vertical axis in Fig. 1 is scaled by $10^{14}$. Oscillating behavior, as in this example, will actually be obtained for both agents and issues as well as for all initial opinions except that of full consensus. The situation is similar for all other critical values of $\tau$.

A main factor that causes $Q$ to have a negative eigenvalue is that the off-diagonal entries of the weight matrices of agent 1 and agent 2 have different signs, i.e., each agent has opposing conceptions about the correlation of the two issues, not only in forming its stubbornness weight but also in its expectation from the other agent through forming the influence weight.

By Theorems 1(i) and 2(ii), if a Nash equilibrium exists, then either $Q$ has at least one negative eigenvalue and the network is in a state of discord, or the network in a state of accord.

\subsection{Two Special Cases}

We now consider two specializations of the result of Theorem 1: first, where agents have pairwise similar views, and second, where all the agents are only connected to one agent called a leader. These two cases come from two extreme assumptions on the structure of the network and lead to more "decoupled" opinion profile expressions for agents. 
Corollary 1 Let $\mathbf{a}=\frac{1}{n} \sum_{j=1}^{n} \mathbf{b}_{j}$ be the average initial opinion.

(i) If $W_{i j}=W_{j i}, \forall i, j \in N$, then a Nash equilibrium exists and is unique. The resulting opinion profile is given by (8).

(ii) If $W_{i i}=F$ and $W_{i j}=G, \forall i, j \in N, i \neq j$, then the Nash equilibrium opinion profile of every agent $i \in N$ is given by

$$
\mathbf{x}_{i}(t)=\mathbf{a}+(F+n G)^{-1}\left\{F+\cosh [\hat{H}(\tau-t)] \cosh (\hat{H} \tau)^{-1} n G\right\}\left(\mathbf{b}_{i}-\mathbf{a}\right),
$$

where $\hat{H}=(F+n G)^{\frac{1}{2}}$ is the real positive definite square root of $F+n G$.

(iii) The opinion profile (10) as $\tau \rightarrow \infty$ is

$$
\mathbf{x}_{i}^{i n f}(t)=\mathbf{a}+(F+n G)^{-1}[F+\exp (-\hat{H} t) n G]\left(\mathbf{b}_{i}-\mathbf{a}\right)
$$

with $\hat{H}=(F+n G)^{\frac{1}{2}}$.

The hypothesis in (ii) is that all agents have identical influence and identical stubbornness weight matrices. This, in effect, means that the agents have the same views of influence from each other. If $G \neq 0$, then every agent is connected to every other agent. This further assumption portrays an ideal situation against which the results obtained in more realistic situations can be compared.

Proof of Corollary 1 If $W_{i j}=W_{j i}$ for all $i, j \in \mathrm{N}$ in (5), then $Q$ is positive definite since, for any $\mathbf{y}_{i}, i \in \mathrm{N}$, computing $\left[\mathbf{y}_{1}^{\prime} \cdots \mathbf{y}_{n}^{\prime}\right] Q\left[\mathbf{y}_{1}^{\prime} \cdots \mathbf{y}_{n}^{\prime}\right]^{\prime}$, we get

$$
\mathbf{y}_{1}^{\prime} W_{11} \mathbf{y}_{1}+\cdots+\mathbf{y}_{n}^{\prime} W_{n n} \mathbf{y}_{n}+\sum_{\forall i, j \in \mathrm{N}, i \neq j}\left(\mathbf{y}_{i}^{\prime}-\mathbf{y}_{j}^{\prime}\right) W_{i j}\left(\mathbf{y}_{i}-\mathbf{y}_{j}\right)>0 .
$$

Here, we used the expression in (5) for $M_{i}$ 's and the hypothesis that $W_{i j}=W_{j i}$ is positive semi-definite for $i \neq j$ and positive definite for $i=j$. It follows by Theorem 1(i) that a unique Nash equilibrium exists, proving (i).

To see (ii), note that, by hypothesis, the matrix $Q$ simplifies to

$$
Q=I_{n} \otimes(F+n G)-\mathscr{I} \otimes G,
$$

where $\mathscr{I}$ is the $n \times n$ matrix of all 1's. It can be verified that $Q=P^{-1} D P$ with $D=$ $\operatorname{diag}[F+n G, \cdots, F+n G, F]$, in which $F+n G$ is repeated $n-1$ times, and

$$
P=\left(\frac{1}{n} \mathscr{I}-K\right) \otimes I_{d}, P^{-1}=\left[\begin{array}{cc}
\mathbf{1}_{n-1}^{\prime} & 1 \\
-I_{n-1} & \mathbf{1}_{n-1}
\end{array}\right] \otimes I_{d},
$$

where $K$ is the $n \times n$ matrix with ones at $(i, i+1)$ locations, for $i=1, \cdots,(n-1)$, and zeros elsewhere. It follows that $Q^{\frac{1}{2}}=P^{-1} D^{\frac{1}{2}} P$ and $\cosh \left(Q^{\frac{1}{2}} t\right)=P^{-1} \cosh \left(D^{\frac{1}{2}} t\right) P$. It is thus a straightforward computation that (8) yields (10), which proves (ii). Finally, the expression in (iii) for the individual profiles in infinite horizon follows by the special structure of $Q$ and $W$ in (9).

Remark 2 (a) Observe from (10) that the two terms that comprise the opinion profile are the constant vector of average initial opinions in the network and the time-dependent term which updates the difference of opinion of agent $i$ on each issue against the average opinion.

(b) It is easy to see that the distinct eigenvalues of $Q$ in this special case is the union of the eigenvalues of $F$ and $F+n G$.

(c) By (11),

$$
\lim _{t \rightarrow \infty} \mathbf{x}_{i}^{\inf }(t)=(F+n G)^{-1}\left(F \mathbf{b}_{i}+n G \mathbf{a}\right)
$$


so that in the long run, agent $i$ reaches at a "convex combination" of his own opinion and the average opinion in the network. How fast the convex combination is attained is determined by the eigenvalues of $\hat{H}$, or equivalently, by the singular values of $F+n G$. A large population (i.e., large $n$ ), strong will (i.e., diagonal entries of $F$ ), and strong harmony (i.e., diagonal entries of $G$ ) will all contribute to fast convergence.

d) A full consensus is never reached on any issue for finite $t$. It is only reached in infinite horizon as $t \rightarrow \infty$ on all issues if and only if the stubbornness matrix tends to zero, in which case the consensus is close to the average initial opinion in the network.

In the network now considered, all the agents are only influenced by one agent called the leader. The leader's opinions on the issues are not influenced by other agents throughout the interval $[0, \tau]$. The neighborhood of the leader (agent 1 ) is $N_{1}=\varnothing$ and the neighborhood of all other agents is $\mathrm{N}_{i}=\{1\}, \forall i \in \mathrm{N} \backslash\{1\}$. It follows that, in this special case, we have

$$
Q=\left[\begin{array}{cccc}
W_{11} & & & \\
-W_{21} & M_{2} & & \\
\vdots & \ddots & \\
-W_{n 1} & & & M_{n}
\end{array}\right],
$$

in which all entries left blank are zero matrices and $M_{i}=W_{i i}+W_{i 1}, \forall i \in \mathrm{N} \backslash\{1\}$.

Corollary 2 (i) For a network where all the agents are only influenced by agent 1, a unique Nash equilibrium exists with the resulting opinion profile given by (8).

(ii) If $W_{11}$ has no common eigenvalue with any $W_{i 1}+W_{i i}$, for $i \in N \backslash\{1\}$, then, in the unique Nash equilibrium that results, the opinion profile of each agent for $t \in[0, \tau]$ is given by $\mathbf{x}_{1}(t)=\mathbf{b}_{1}$ and

$$
\mathbf{x}_{i}(t)=\left(W_{i i}+W_{i 1}\right)^{-1}\left\{W_{i i} \mathbf{b}_{i}+W_{i 1} \mathbf{b}_{1}+\cosh \left[\hat{H}_{i}(\tau-t)\right] \cosh \left(\hat{H}_{i} \tau\right)^{-1} W_{i 1}\left(\mathbf{b}_{i}-\mathbf{b}_{1}\right)\right\}
$$

for $i \in N \backslash\{1\}$, where $\hat{H}_{i}=\left(W_{i i}+W_{i 1}\right)^{\frac{1}{2}}$ is the real positive definite square root.

(iii) The opinion profile as $\tau \rightarrow \infty$ is

$$
\mathbf{x}_{i}^{i n f}(t)=\left(W_{i i}+W_{i 1}\right)^{-1}\left[W_{i i} \mathbf{b}_{i}+W_{i 1} \mathbf{b}_{1}+\exp \left(-\hat{H}_{i} t\right) W_{i 1}\left(\mathbf{b}_{i}-\mathbf{b}_{1}\right)\right] .
$$

Proof Note that the lower block triangular $Q$ of (12) is nonsingular with positive eigenvalues by positive definiteness of its diagonal blocks. Hence, $f_{Q}(\tau)$ is nonsingular and (i) holds by Theorem 1(i). By the hypothesis in (ii), a solution $X$ to the Sylvester equation $X \hat{W}_{1}-\hat{W}_{2} X=$ $\hat{W}_{3}$ exists, which implies that

$$
Q=\left[\begin{array}{cc}
\hat{W}_{1} & 0 \\
-\hat{W}_{3} & \hat{W}_{2}
\end{array}\right]=\left[\begin{array}{ll}
I & 0 \\
X & I
\end{array}\right]^{-1}\left[\begin{array}{cc}
\hat{W}_{1} & 0 \\
0 & \hat{W}_{2}
\end{array}\right]\left[\begin{array}{ll}
I & 0 \\
X & I
\end{array}\right],
$$

where $\hat{W}_{1}=W_{11}, \hat{W}_{2}=\operatorname{diag}\left[W_{21}+W_{22}, \cdots, W_{n 1}+W_{n n}\right]$ and $\hat{W}_{3}=\left[W_{21}^{\prime}, \cdots, W_{n 1}^{\prime}\right]^{\prime}$. It follows that

$$
H=\left[\begin{array}{ll}
I & 0 \\
X & I
\end{array}\right]^{-1}\left[\begin{array}{cc}
\hat{W}_{1}^{\frac{1}{2}} & 0 \\
0 & \hat{W}_{2}^{\frac{1}{2}}
\end{array}\right]\left[\begin{array}{ll}
I & 0 \\
X & I
\end{array}\right]
$$

is a real square root of $Q$ with positive eigenvalues. This gives an expression for $\cosh (H t)$ in terms of $\hat{H}_{i}=\left(W_{i i}+W_{i 1}\right)^{\frac{1}{2}}$ for $i=2, \cdots, n$ and proves (ii) by Theorem 1(ii). Limits in 
(iii) easily follow by the expression (13). (The square root $\hat{W}_{1}^{\frac{1}{2}}=W_{11}^{\frac{1}{2}}$, which appears in $H$, cancels out in the final expressions (13).)

Remark 3 (a) The sufficient condition of Corollary 2(ii) is satisfied for almost all choices of weight matrices. This condition ensures that a real square root of (12) with positive eigenvalues exists.

(b) The expression (13) has again two parts. The constant first part

$$
\left(W_{i i}+W_{i 1}\right)^{-1}\left(W_{i i} \mathbf{b}_{i}+W_{i 1} \mathbf{b}_{1}\right)
$$

can be viewed as a weighted convex combination of the initial opinion vectors of the leader and agent $i$. The dynamic second part updates their difference in opinions in the interval $[0, \tau]$.

(c) By Corollary 2(iii), the opinion vector reached as $t \rightarrow \infty$ by agent $i$ is a convex combination of its own opinions and those of the leader. The difference $\mathbf{x}_{i}^{\inf }(t)-\mathbf{x}_{1}^{\inf }(t)$ indicates that agent $i$ will be of the same opinion as the leader as $t \rightarrow \infty$ if and only if it is not at all stubborn, i.e., $W_{i i}$ is close to 0 .

(d) The larger the all eigenvalues of $\hat{H}_{i}$ are, the faster will be the partial consensus that will be reached on the issues in the long run.

(e) The results in Corollary 2 extend to more general network structures of star or acyclic graph topologies but require stronger assumptions. For example, a lower block triangular $Q$ having nonzero influence weights $W_{i j}, i>j$, requires that eigenvalues of every pair $M_{i}, M_{j}$ are distinct.

\section{An Application Example}

This section provides a rationale for choosing the entries of the weight matrices and illustrates some consequences of their choices in an example of two hypothetical political parties.

\subsection{On the Entries of Weight Matrices}

It is safe to assume in a social network that the frequency of interaction is higher between agents who are characteristically similar to each other [5] which is known as homophily [26]. Hence, agents usually tend to appreciate and get influenced by those who are similar in ethnicity, geography, etc. Through interactions, the agents are usually positively influenced by each other, but negative influences [21] may also arise through antagonistic interactions. Such antagonistic interactions $[3,39,42]$ may cause them to move away from each other in opinions. Antagonistic interactions arise in our model from the reaction of agents, who take into account that some of the issues may be interdependent or correlated. One assumption we adopt is that an agent will boycott those who hold inconsistent opinions on issues that it considers to be correlated. Hence, agents approve or boycott others based on the consistency of their opinions on certain social issues [43].

The rationale in the choice of a weight matrix $W_{i j}$ by agent $i$ must be based on the main motive of minimizing the cost (1). Consider an influence matrix $W_{i j}, i \neq j$, then the first term in the cost $\left(\mathbf{x}_{i}-\mathbf{x}_{j}\right)^{\prime} W_{i j}\left(\mathbf{x}_{i}-\mathbf{x}_{j}\right)$ can be written as 


$$
\sum_{k=1}^{d}\left[w_{i j}^{k k}\left(x_{i}^{k}-x_{j}^{k}\right)^{2}+\sum_{\substack{l=1 \\ l \neq k}}^{d}\left(w_{i j}^{k l}+w_{i j}^{l k}\right)\left(x_{i}^{k}-x_{j}^{k}\right)\left(x_{i}^{l}-x_{j}^{l}\right)\right]
$$

where the summands due to diagonal and off-diagonal entries of $W_{i j}$ are separately displayed. A choice $w_{i j}^{k k}>0$ indicates that $i$ is willing to approach the opinion of $j$ on issue $k$ in the interval $(0, \tau]$ because minimization of the cost requires keeping $\left|x_{i}^{k}-x_{j}^{k}\right|$ small. By contrast, $w_{i j}^{k k}<0$ would have required a divergence of opinions. However, by Assumption 1, we postulated that $i$ chooses $w_{i j}^{k k}>0$ for every issue $k$.

A choice $w_{i j}^{k l}=w_{i j}^{l k}$, i.e., symmetry, which is also postulated by Assumption 1, ensures that the summands resulting from these two off-diagonal entries have the same effect on the cost. If $w_{i j}^{k l}$ is nonzero, then the term $2 w_{i j}^{k l}\left(x_{i}^{k}-x_{j}^{k}\right)\left(x_{i}^{l}-x_{j}^{l}\right)$ in the cost reflects the opinion of $i$ on the correlation between issues $k$ and $l$ throughout the interval $(0, \tau]$. A choice $w_{i j}^{k l}>0$ indicates that the two issues are believed by agent $i$ to be positively correlated so that whenever the product $\left(x_{i}^{k}-x_{j}^{k}\right)\left(x_{i}^{l}-x_{j}^{l}\right)$ is positive ( $i$ and $j$ hold differing but consistent opinions on both issues), minimization of the cost will require that $i$ approaches to the viewpoint of $j$ on both issues. Similarly, a choice $w_{i j}^{k l}<0$ indicates that the two issues are believed by agent $i$ to be negatively correlated so that whenever the product $\left(x_{i}^{k}-x_{j}^{k}\right)\left(x_{i}^{l}-x_{j}^{l}\right)$ is negative ( $i$ and $j$ hold differing and inconsistent opinions on the two issues), minimization of the cost will again require that $i$ approaches to the viewpoint of $j$ on both issues. In all the other cases, minimization of the cost will require $i$ to move away from some or all of the opinions of $j$. We can thus say that a positive off-diagonal $k l$ th entry of $W_{i j}$ indicates that agent $i$ believes issues $k$ and $l$ to be positively correlated; a negative entry, on the other hand, indicates a belief of negative correlation. (Here, we have adopted an agent $i$-centered interpretation of the choice of entries in $W_{i j}$. It is equally legitimate to adopt a $j$-centered viewpoint.) Concerning an influence weight $W_{i j}$, we have only supplied a support for the assumptions that it is symmetric with positive diagonal entries. In Assumption 1, we further require that it is positive semi-definite. (This is of course only to ensure that a technically easy to handle convex cost (1) is obtained.)

The interpretation of entries of a stubbornness weight matrix $W_{i i}$ follows an entirely similar rationale, by simply replacing the agent $j$ in the narrative above by agent $i$ 's "initial self." The stubbornness on an issue is penalized by the diagonal entries and the notion of correlation of two issues by the off-diagonal entries.

\subsection{An Example of a Network with Two Political Parties}

Current debates of two (hypothetical) political parties, party-A and party-B, are about two correlated issues. Issue 1 is "private ownership of guns" and issue 2 is "death penalty." Party-A holds a positive opinion on issue 1, i.e., private ownership of guns should be allowed for self-defensive purposes, but holds an indifferent opinion on issue 2, i.e., eradication of death penalty right now is not a major concern. So, we can represent the initial opinion of party-A by a vector $\mathbf{b}_{1}=\left[\begin{array}{ll}1 & 0\end{array}\right]^{\prime}$. However, party-A has a conception that these two issues are negatively correlated, i.e., private ownership of guns does not entail the crimes which are punishable by death. Party-B differs from party-A and holds opinions that private ownership of guns should be restricted and death penalty should be eradicated, and considers both issues to be positively correlated. Therefore, the initial opinions of party-B can be represented by $\mathbf{b}_{2}=\left[\begin{array}{ll}-1 & -1\end{array}\right]^{\prime}$. 


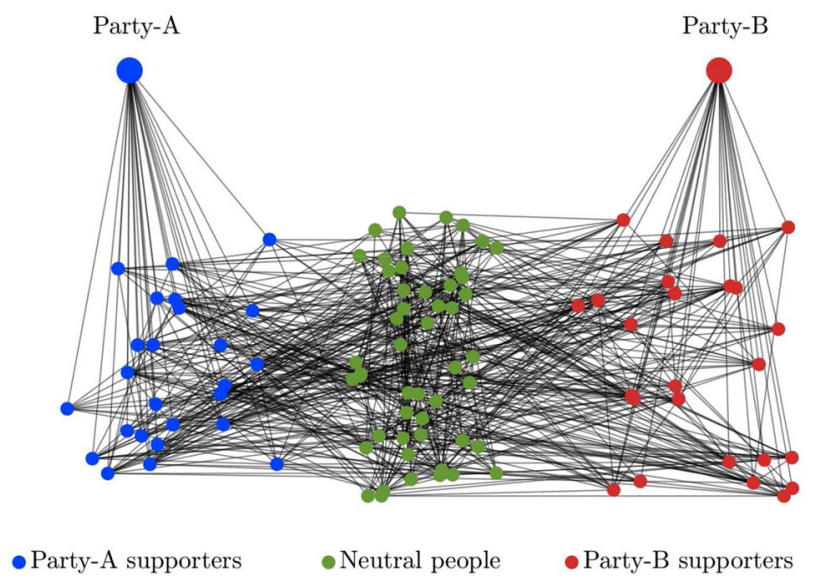

Fig. 2 Network topology

We assume both parties propose reasonable arguments in support of their opinions so that they have some supporters, Supporters-A and Supporters-B, assumed to be of the same number, e.g., 25. Supporters-A hold initial opinions in the intervals $(0.5,1.5)$ and $(-0.5,0.5)$ on the two issues, respectively, and Supporters-B in the interval $(-1.5,-0.5)$ on both issues. In addition, there are 50 neutral people, who hold initial opinions in the intervals $(-0.5,0.5)$ and $(-1.5,0.5)$ on the two issues, respectively. We thus have total number of agents $n=102$ by also counting the two parties as agents.

The weight matrices are chosen as follows:

$$
W_{i i}=\left\{\begin{array}{l}
W_{A A}, \text { if } i \in \text { Party-A } \cup \text { Supporters-A, } \\
W_{B B}, \text { if } i \in \text { Party-B } \cup \text { Supporters-B. }
\end{array}\right.
$$

For $i \neq j$, we have

$$
W_{i j}=\left\{\begin{array}{l}
W_{A j}, \text { if } i \in \text { Party-A } \cup \text { Supporters-A, } \\
W_{B j}, \text { if } i \in \text { Party-B } \cup \text { Supporters-B. }
\end{array}\right.
$$

If $i \in$ Neutral people, then we choose $W_{i i}$ and $W_{i j}$ randomly from $\left\{W_{A A}, W_{B B}\right\}$ and $\left\{W_{A j}, W_{B j}\right\}$, respectively, in the same order, i.e., $W_{A A}$ (resp., $W_{B B}$ ) is paired with $W_{A j}$ (resp., $W_{B j}$ ). The agents are connected through a network topology as shown in Fig. 2. The game is considered under three different sets of weights and in a duration of length $\tau=30$. In the following, we present three cases that may occur under Nash equilibrium based on the choice of weight matrices.

\subsubsection{Case 1 (Opinion Spread)}

The stubbornness weight matrices $W_{A A}$ and $W_{B B}$ and influence matrices $W_{A j}$ and $W_{B j}$ are 



Fig. 3 Opinion spread (Case 1)

$$
\begin{aligned}
& W_{A A}=\left[\begin{array}{rr}
1 & -1 \\
-1 & 2
\end{array}\right], W_{B B}=\left[\begin{array}{ll}
2 & 1 \\
1 & 1
\end{array}\right], \\
& W_{A j}=\left[\begin{array}{rr}
1 & -1 \\
-1 & 1
\end{array}\right], W_{B j}=\left[\begin{array}{ll}
1 & 1 \\
1 & 1
\end{array}\right] .
\end{aligned}
$$

In the influence matrices, the correlation terms (off-diagonal entries of $W_{A j}$ and $W_{B j}$ ) have the same magnitudes as the influence terms (diagonal entries). That means that agents consider the importance on the consistency of their neighbors' opinions equal to their influence. Therefore, if some agent finds its neighbors' opinions inconsistent according to its conception of correlation, it is equally good for its motive to move away from their opinions rather than form (partial) consensus. Hence, in Fig. 3, we see a spread of opinion trajectories.

\subsubsection{Case 2 (Partial Consensus)}

We keep the same $W_{A A}, W_{B B}$ but strengthen the influence terms of the influence matrices in (14) as

$$
W_{A j}=\left[\begin{array}{cc}
1.25 & -1 \\
-1 & 1.25
\end{array}\right], W_{B j}=\left[\begin{array}{cc}
1.25 & 1 \\
1 & 1.25
\end{array}\right] .
$$

Now that the magnitudes of the influence terms (diagonals) in the influence matrices are greater than the correlation terms, the agents are more inclined to form consensus and they somehow neglect inconsistencies of their neighbors. This results in Fig. 4 that illustrates a partial consensus at $\tau$.

\subsubsection{Case 3 (Oscillations)}

Here, our purpose is to demonstrate oscillatory opinion trajectories and the matrices in (14) need be chosen such that $Q$ has a negative eigenvalue. The choice

$$
\begin{aligned}
& W_{A A}=\left[\begin{array}{rr}
0.78 & -0.84 \\
-0.84 & 2.81
\end{array}\right], W_{B B}=\left[\begin{array}{ll}
1.95 & 2.15 \\
2.15 & 3.85
\end{array}\right], \\
& W_{A j}=3.36\left[\begin{array}{rr}
1 & -1 \\
-1 & 1
\end{array}\right], \quad W_{B j}=4.55\left[\begin{array}{ll}
1 & 1 \\
1 & 1
\end{array}\right] .
\end{aligned}
$$

results in a matrix $Q$ with a real negative eigenvalue at $-r^{2}=-0.0761$ and gives oscillatory trajectories of period $2 \pi / r=22.8859$, as shown in Fig. 5 . The trajectories oscillate and do not 

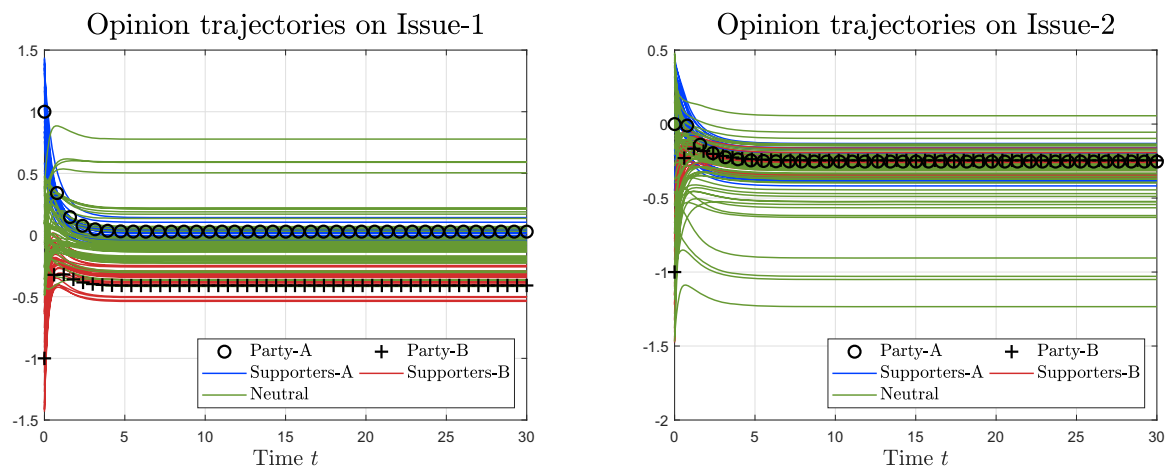

Fig. 4 Partial consensus (Case 2)

converge, i.e., agents are hesitant as to which opinions to maintain. A necessary cause of this behavior is the opposite conceptions about the correlations of the issues in the society. This is not sufficient by itself as the previous two cases also have the same pattern of correlation but are without oscillations. The sufficient condition of having a real negative eigenvalue for $Q$ also requires a thin balance between the repulsion caused by opposing correlation terms and the attraction caused by the influence terms. The agents are captured between two pressures to update their opinions. On the one hand their conceptions of correlation of the issues are diametrically opposite to some of their neighbors while on the other hand, they are also susceptible to be influenced by their neighbors, who might be the supporters of other party or neutral people. (Keep in mind that all agents maintain their conception of correlation between issues 1 and 2 also in their stubbornness weight matrices.) On issue-1, in Fig. 5, party-A and supporters-A struggle to form consensus with party-B and supporters-B, who on the other hand struggle to stay consistent about their conception of positive correlation between the issues. In this struggle, agents end up oscillating in a synchronous way. On issue-2, however, party-A and their supporters keep their opinions roughly constant and party-B and supporters oscillate as a compromise between consistency and consensus.
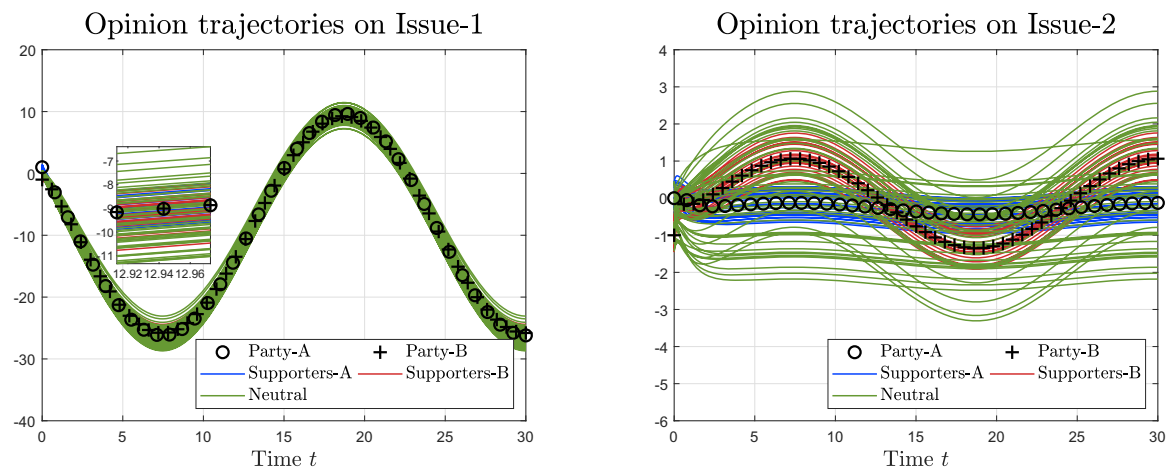

Fig. 5 Oscillatory opinion trajectories (Case 3) 


\section{Concluding Remarks}

Our model-based investigation of opinion dynamics on multiple issues utilized game theory and provided necessary and sufficient conditions for the existence of a unique Nash equilibrium. It turns out that a Nash equilibrium may not exist in certain information structures and/or certain values for the weights used in forming the individual objective functions. Interestingly, a state of discord, in which there are persisting oscillatory opinion dynamics, can also be a Nash equilibrium. Such an equilibrium, nevertheless, is an exceptional state of harmony.

More often than not, a Nash equilibrium that results is a state of accord in the network and can be viewed as a partial consensus. Such Nash equilibrium may arise from different characteristics of individuals such as a democratically acquired similarity in views or assenting to an authority in the society.

Theorems 1 and 2 demonstrate that negative eigenvalues of $Q$ are bad for convergence of opinions toward a consensus. Consistently, we further learned from Corollaries 1 and 2 that larger the positive eigenvalues of $Q$, faster is the convergence of opinion profiles to a (partial) consensus. We have gained some insight into the network structures and weight matrix characteristics that would result in the wide spectrum from negative eigenvalues in $Q$ to large positive ones:

i) Any unique opinion profile that results by an initial opinion vector $\mathbf{b}$ can be decomposed into parts that are triggered by two components of $\mathbf{b}$. One part is due to the component of $\mathbf{b}$ in the null space of $Q-W$, which is never trivial for any network structure and contains at least the full consensus. The null space consists of constant Nash profiles $\mathbf{b}$ that are composed of differences of initial opinions $\mathbf{b}_{i}-\mathbf{b}_{j}$ "filtered by the influence weights." (See Remark 1(b) and (c).)

ii) The Nash equilibrium that is a state of discord can only arise in a truly multiple issue game. A sufficient condition for such oscillatory profiles to occur is, technically, a $Q$ matrix with a negative eigenvalue. This is a result of two pressures agents undergo, moving away from the opinion of neighbors' having different notions of correlation among issues and approaching the opinion of the same neighbors due to the built-in motives to be positively influenced.

iii) The Nash equilibrium fails to exist in some critical lengths of finite duration $\tau$, in which the game is played, but not in other values, because the magnitude of the oscillations in discord opinion dynamics is proportional to $2 / \cos (r \tau)$. It becomes infinite only at odd multiples of $\tau=\pi /(2 r)$. Growing oscillations without bound occur when the positive influences are insufficient to check the pressure to move away from the contested opinion.

Our investigation of dynamic opinion games will continue to explore different classes of objective (cost) functionals that may represent more sophisticated motives attributed to agents in a society.

Acknowledgements This work is supported by the Science and Research Council of Turkey (TÜBITAK) under the project EEEAG-114E270. We would like to thank Dr. Aykut Yildız for his contributions at the earlier stages of this project. We would also like to thank the editor and the anonymous reviewer for their invaluable suggestions to improve the paper. 


\section{Appendix}

\section{The Game of Opinion Dynamics is of the Linear Quadratic Type}

To show that (2) is of the linear quadratic type [6, Definition 6.5], we consider

$$
\mathbf{z}_{i}=\left[\begin{array}{llllll}
\Delta_{i 1}^{\prime} & \ldots & \Delta_{i(i-1)}^{\prime} & \Delta_{i i}^{\prime} & \ldots & \Delta_{i n}^{\prime}
\end{array}\right]^{\prime} \in \mathbb{R}^{n d},
$$

where

$$
\Delta_{i j}:=\left\{\begin{array}{l}
\mathbf{x}_{i}-\mathbf{x}_{j}, \text { if } i \neq j \\
\mathbf{x}_{i}-\mathbf{b}_{i}, \text { if } i=j
\end{array}\right.
$$

for $i, j=1, \ldots, n$. Then, we have $\mathbf{z}=\left[\begin{array}{lll}\mathbf{z}_{1}^{\prime} & \ldots & \mathbf{z}_{n}^{\prime}\end{array}\right]^{\prime} \in \mathbb{R}^{n^{2} d}$ as the state vector, which satisfies

$$
\dot{\mathbf{z}}=\sum_{i=1}^{n} B_{i} \mathbf{u}_{\mathbf{i}},
$$

where $B_{i} \in \mathbb{R}^{n^{2} d \times d}$ is given as

$$
B_{i}=\left[-\left(\mathbf{e}_{i} \otimes I_{d}\right)^{\prime} \cdots-\left(\mathbf{e}_{i} \otimes I_{d}\right)^{\prime}\left(\mathbf{1}_{n} \otimes I_{d}\right)^{\prime}-\left(\mathbf{e}_{i} \otimes I_{d}\right)^{\prime} \cdots-\left(\mathbf{e}_{i} \otimes I_{d}\right)^{\prime}\right]^{\prime},
$$

where the matrix block $\mathbf{1}_{n} \otimes I_{d}$ is the $i$ th block and $\mathbf{e}_{i}=\left[\begin{array}{lllll}0 \ldots & \ldots & 1 & 0 \ldots 0\end{array}\right]^{\prime} \in \mathbb{R}^{n}$ is the standard $i$ th basis vector of $\mathbb{R}^{n}$.

The cost functional (1) can now be written as

$$
\mathscr{J}_{i}\left(\mathbf{z}, \mathbf{u}_{i}\right)=\frac{1}{2} \int_{0}^{\tau}\left(\mathbf{z}^{\prime} L_{i} \mathbf{z}+\mathbf{u}_{i}^{\prime} \mathbf{u}_{i}\right) d t,
$$

where $L_{i}=H_{i} \otimes K_{i} \in \mathbb{R}^{n^{2} d \times n^{2} d}$ with $H_{i} \in \mathbb{R}^{n \times n}$ whose $j k$ th entry, for $j, k=1, \ldots, n$, is defined as

$$
\left[H_{i}\right]_{j k}=\left\{\begin{array}{l}
1, \text { if } j=k=i \\
0, \text { otherwise }
\end{array}\right.
$$

and $K_{i}=\operatorname{diag}\left[W_{i 1}, \ldots, W_{i n}\right] \in \mathbb{R}^{n d \times n d}$. From the above formulation, it can be seen that the game described by (15) and (17) is a linear quadratic differential game. By Assumption $1, L_{i}$ is positive semi-definite, which gives that (17), equivalently (1), is a strictly convex function of $\mathbf{u}_{i}(t)$. Hence, the Nash equilibrium, if it exists, is unique [6, Proof of Theorem 6.12]. Thus, we can skip a more familiar approach of coupled Riccati differential equations, which involve manipulations with large sparse matrices like $L_{i}$ above.

\section{Proof of Theorem 1 Completed}

The necessary conditions ([6, Theorem 6.11], [27, Chapter 5]) for the existence of a minimum of the cost function (1) are

$$
\begin{aligned}
& \mathbf{u}_{i}^{*}(t)=\arg \min _{\mathbf{u}_{i} \in \mathrm{S}_{i}} \mathscr{H}_{i}\left(\mathbf{p}_{i}, \mathbf{x}, \mathbf{b}_{i}, \mathbf{u}_{i}\right), \\
& \dot{\mathbf{x}}_{i}(t)=\frac{\partial \mathscr{H}_{i}}{\partial \mathbf{p}_{i}}, \quad \dot{\mathbf{p}}_{i}(t)=-\frac{\partial \mathscr{H}_{i}}{\partial \mathbf{x}_{i}}, \\
& \mathbf{x}_{i}(0)=\mathbf{b}_{i}, \quad \mathbf{p}_{i}(\tau)=0, \quad i \in \mathrm{N},
\end{aligned}
$$


where $\mathbf{p}_{i}:[0, \tau] \rightarrow \mathbb{R}^{n d}$ is a costate function and $\mathscr{H}_{i}$ is the Hamiltonian given by

$$
\mathscr{H}_{i}=\frac{1}{2}\left\{\left[\sum_{j \in \mathrm{N}_{i}} \Delta_{i j}^{\prime} W_{i j} \Delta_{i j}\right]+\Delta_{i i}^{\prime} W_{i i} \Delta_{i i}+\mathbf{u}_{i}^{\prime} \mathbf{u}_{i}\right\}+\mathbf{p}_{i}^{\prime} \mathbf{u}_{i} .
$$

Let $\mathbf{p}=\left[\begin{array}{lll}\mathbf{p}_{1}^{\prime} & \cdots & \mathbf{p}_{n}^{\prime}\end{array}\right]^{\prime}$ and note that the combined state and costate equations are, by (18),

$$
\left[\begin{array}{c}
\dot{\mathbf{x}} \\
\dot{\mathbf{p}}
\end{array}\right]=\left[\begin{array}{cc}
0 & -I \\
-Q & 0
\end{array}\right]\left[\begin{array}{l}
\mathbf{x} \\
\mathbf{p}
\end{array}\right]+\left[\begin{array}{cc}
0 & 0 \\
W & 0
\end{array}\right]\left[\begin{array}{c}
\mathbf{b} \\
\mathbf{p}(0)
\end{array}\right],
$$

which has the solution of the form

$$
\left[\begin{array}{l}
\mathbf{x} \\
\mathbf{p}
\end{array}\right]=(\Phi(t)+\Psi(t) B)\left[\begin{array}{c}
\mathbf{b} \\
\mathbf{p}(0)
\end{array}\right],
$$

where

$$
\begin{aligned}
\Phi(t) & =e^{A t}=\left[\begin{array}{ll}
\phi_{11}(t) & \phi_{12}(t) \\
\phi_{21}(t) & \phi_{22}(t)
\end{array}\right], \\
\Psi(t) & =\int_{0}^{t} e^{A(t-\mu)} d \mu=\left[\begin{array}{ll}
\psi_{11}(t) & \psi_{12}(t) \\
\psi_{21}(t) & \psi_{22}(t)
\end{array}\right], \\
A & =\left[\begin{array}{cc}
0 & -I \\
-Q & 0
\end{array}\right], \quad B=\left[\begin{array}{cc}
0 & 0 \\
W & 0
\end{array}\right] .
\end{aligned}
$$

Note that

$$
\begin{aligned}
\Phi(t) & =\mathscr{L}^{-1}\left\{(s I-A)^{-1}\right\} \\
& =\mathscr{L}^{-1}\left\{\left[\begin{array}{cc}
s\left(s^{2} I-Q\right)^{-1} & -\left(s^{2} I-Q\right)^{-1} \\
-Q\left(s^{2} I-Q\right)^{-1} & s\left(s^{2} I-Q\right)^{-1}
\end{array}\right]\right\} .
\end{aligned}
$$

The state transition matrix $\Phi(t)$ and the matrix $\Psi(t)$ are calculated using the formal power series in $s^{-1}$ of each block in (20) and, with (3) in view, are given by

$$
\begin{gathered}
\Phi(t)=\left[\begin{array}{ll}
\phi_{11}(t) & \phi_{12}(t) \\
\phi_{21}(t) & \phi_{22}(t)
\end{array}\right]=\left[\begin{array}{cc}
f_{Q}(t) & -g_{Q}(t) \\
-Q g_{Q}(t) & f_{Q}(t)
\end{array}\right], \\
\Psi(t)=\left[\begin{array}{ll}
\psi_{11}(t) & \psi_{12}(t) \\
\psi_{21}(t) & \psi_{22}(t)
\end{array}\right]=\left[\begin{array}{cc}
g_{Q}(t) & -h_{Q}(t) \\
-Q h_{Q}(t) & g_{Q}(t)
\end{array}\right] .
\end{gathered}
$$

From (19), we have

$$
\mathbf{x}(t)=\left[\phi_{11}(t)+\psi_{12}(t) W\right] \mathbf{b}+\phi_{12}(t) \mathbf{p}(0) .
$$

Also,

$$
\mathbf{p}(t)=\left[\phi_{21}(t)+\psi_{22}(t) W\right] \mathbf{b}+\phi_{22}(t) \mathbf{p}(0) .
$$

Evaluating at $t=\tau$ and employing the boundary condition $\mathbf{p}(\tau)=0$, we obtain

$$
\phi_{22}(\tau) \mathbf{p}(0)=-\left[\phi_{21}(\tau)+\psi_{22}(\tau) W\right] \mathbf{b},
$$

or substituting the expressions in terms of $f_{Q}(t), g_{Q}(t), h_{Q}(t)$ above,

$$
f_{Q}(\tau) \mathbf{p}(0)=g_{Q}(\tau)(Q-W) \mathbf{b}
$$

and

$$
\mathbf{x}(t)=\mathbf{b}+h_{Q}(t)(Q-W) \mathbf{b}-g_{Q}(t) \mathbf{p}(0) .
$$


If $f_{Q}(\tau)$ is nonsingular, then a unique solution $\mathbf{p}(0)$ to $(21)$ exists for all $\mathbf{b}$. If $f_{Q}(\tau)$ is singular, then there is a negative eigenvalue $-r^{2}$ of $Q$. Let $\mathbf{v}$ be a left eigenvector associated with $-r^{2}$ and note that

$$
\mathbf{v}^{\prime} Q=-r^{2} \mathbf{v}^{\prime}, \mathbf{v}^{\prime} f_{Q}(\tau)=\cos (r \tau) \mathbf{v}^{\prime}, \mathbf{v}^{\prime} g_{Q}(\tau)=r^{-1} \sin (r \tau) \mathbf{v}^{\prime}
$$

It follows, by multiplying (21) by $\mathbf{v}^{\prime}$ on the left, that $\cos (r \tau) \mathbf{v}^{\prime} \mathbf{p}(0)=-r^{-1} \sin (r \tau) \mathbf{v}^{\prime}\left(r^{2} I+\right.$ $W) \mathbf{b}$. If $r \tau$ is an odd multiple of $\pi / 2$, then the left-hand side is zero and the right-hand side is nonzero for at least $\mathbf{b}=\mathbf{v}$ as $r^{2} I+W$ is symmetric, positive definite. It follows that $f_{Q}(\tau)$ must be nonsingular for a solution to exist for all $\mathbf{b}$.

Summarizing, in order to be able to solve (21) uniquely for $\mathbf{p}(0)$ for any initial state b it is necessary and sufficient that $f_{Q}(\tau)$ is invertible. This establishes the necessity and sufficiency of the condition (C1) of Theorem 1(i) for the existence of a unique Nash solution. If $f_{Q}(\tau)$ is invertible, we then obtain $\mathbf{p}(0)=f_{Q}(\tau)^{-1} g_{Q}(\tau)(Q-W) \mathbf{b}$. Substituting in $\mathbf{x}(t)=\left[f_{Q}(t)-h_{Q}(t) W\right] \mathbf{b}-g_{Q}(t) \mathbf{p}(0)$, we obtain

$$
\mathbf{x}(t)=\left[f_{Q}(t)-h_{Q}(t) W\right] \mathbf{b}-g_{Q}(t) f_{Q}(\tau)^{-1} g_{Q}(\tau)(Q-W) \mathbf{b},
$$

which gives (7) upon employing $f_{Q}(t)=I+Q h_{Q}(t)$ and noting again that functions of $Q$ commute. Taking the derivative with respect to $t$ and using $\frac{d}{d t} h_{Q}(t)=g_{Q}(t), \frac{d}{d t} g_{Q}(t)=$ $f_{Q}(t)$, we also obtain (6). This completes the proof of Theorem 1 .

Remark 4 (Non-unique equilibria) Here, we briefly characterize non-unique Nash profiles that may arise. Suppose $f_{Q}(\tau)$ is singular, so that $(\mathrm{C} 2)$ of Theorem 1 holds. Then, with $J_{Q}=P^{-1} Q P$ denoting the Jordan form of $Q$, Eq. (21) is equivalent to

$$
\left[\begin{array}{cc}
f_{J_{r}}(\tau) & 0 \\
0 & f_{J}(\tau)
\end{array}\right]\left[\begin{array}{l}
\mathbf{p}_{1} \\
\mathbf{p}_{2}
\end{array}\right]=\left[\begin{array}{l}
G_{1}(\tau) \\
G_{2}(\tau)
\end{array}\right](Q-W) \mathbf{b}
$$

where $\operatorname{diag}\left[J_{r}, J\right]=J_{Q},\left[G_{1}(\tau)^{\prime} G_{2}(\tau)^{\prime}\right]^{\prime}=P^{-1} g_{Q}(\tau),\left[\mathbf{p}_{1}^{\prime} \mathbf{p}_{2}^{\prime}\right]^{\prime}=\mathbf{p}(0)$ with $J_{r}$ denoting the Jordan blocks associated with the negative eigenvalue $-r^{2}$ of $Q$; and $J$, associated with the other eigenvalues. It follows that, if $\tau$ is an odd multiple of $\pi /(2 r)$, then $f_{J_{r}}(\tau)$ is singular and $f_{J}(\tau)$ is nonsingular. In this case, $\mathbf{p}(0)$ and $\mathbf{b}$ satisfy (21) if and only if $\mathbf{p}_{1}$ is in the null space of $f_{J_{r}}(\tau), \mathbf{p}_{2}=\left[f_{J}(\tau)\right]^{-1} G_{2}(Q-W) \mathbf{b}$, and $G_{1}(Q-W) \mathbf{b}=0$. (It is easy to see that $\mathbf{p}_{1}$ is in the null space of $f_{J_{r}}(\tau)$ if and only if $\mathbf{v}^{\prime} f_{Q}(\tau) \mathbf{p}(0)=0$ for all left eigenvectors of $Q$ associated with $-r^{2}$.) Since the null space of $f_{J_{r}}(\tau)$ for $\tau$ that are odd multiples of $\pi /(2 r)$ is nontrivial, $\mathbf{p}_{1} \neq 0$ results in infinitely many different $\mathbf{x}(t)$ in (22). Among $\mathbf{b}$ such that $G_{1}(Q-W) \mathbf{b}=0$, one can distinguish between $\mathbf{b}=\tilde{\mathbf{b}}$ that are in the null space of $Q-W$ and those with nonzero components $\hat{\mathbf{b}}$ in the range space of $(Q-W)^{\prime}$, see Remark 1(c). In the first case, the choice $\mathbf{p}_{2}=0$ is necessary and for $\mathbf{p}_{1}=0,(22)$ gives the constant profile $\mathbf{x}(t)=\mathbf{b}$, while any $\mathbf{p}_{1} \neq 0$ in the null space of $f_{J_{r}}(\tau)$ gives $\mathbf{x}(t)=\mathbf{b}-g_{Q}(t) \mathbf{p}(0)$ as another profile that is not constant since $g_{Q}(t)$ is nonsingular for almost all $t$, by Remark $1(\mathrm{~d})$. In the second, $(Q-W) \mathbf{b}=(Q-W) \hat{\mathbf{b}}$ gives $\mathbf{p}_{2}=\left[f_{J}(\tau)\right]^{-1} G_{2}(Q-W) \hat{\mathbf{b}}$ and there are again infinitely many profiles (22) that result from different choices of $\mathbf{p}_{1}$ in the null space of $f_{J_{r}}(\tau)$. This characterizes all non-unique solutions to (21). We emphasize that constant profiles that are in the null space of $Q-W$ exist whether $f_{Q}(\tau)$ is singular or not, but they lead to unique opinion profiles only if $f_{Q}(\tau)$ is nonsingular. 


\section{Proof of Theorem 2(iii)}

By Section 2.1, $H_{p}=H_{+}-H_{-}$for a square root $H=H_{+}+H_{-}$of $Q$ so that

$$
\begin{aligned}
& \lim _{\tau \rightarrow \infty} \cosh [H(\tau-t)] \cosh (H \tau)^{-1} \\
& =\lim _{\tau \rightarrow \infty}\left\{\begin{array}{l}
\left\{\exp \left[\left(H_{+}+H_{-}\right)(\tau-t)\right]+\exp \left[-\left(H_{+}+H_{-}\right)(\tau-t)\right\}\right. \\
\times\left\{\exp \left[\left(H_{+}+H_{-}\right) \tau\right]+\exp \left[-\left(H_{+}+H_{-}\right) \tau\right]\right\}^{-1}
\end{array}\right\} \\
& =\exp \left(-H_{+} t\right) \exp \left(H_{-} t\right) \lim _{\tau \rightarrow \infty}\left\{\begin{array}{l}
\left\{\exp \left[2 H_{-}(\tau-t)\right]+\exp \left[-2 H_{+}(\tau-t)\right]\right\} \\
\times\left[\exp \left(2 H_{-} \tau\right)+\exp \left(-2 H_{+} \tau\right)\right]^{-1}
\end{array}\right\} \\
& =\exp \left(-H_{p} t\right)
\end{aligned}
$$

where the last equality follows by

$$
\lim _{\tau \rightarrow \infty}\left\{\exp \left[2 H_{-}(\tau-t)\right]+\exp \left[-2 H_{+}(\tau-t)\right]\right\}=I
$$

and by

$$
\lim _{\tau \rightarrow \infty}\left[\exp \left(2 H_{-} \tau\right)+\exp \left(-2 H_{+} \tau\right]\right]=I .
$$

Therefore, applying the limit to (8), the expression for $\mathbf{x}^{\inf }(t)$ in (9) is obtained. This completes the proof of Theorem 2.

Remark 5 (Connection with the infinite horizon game) If $Q$ is free of any real negative eigenvalue, then it has real square roots $H$, all of which have eigenvalues with nonzero real parts including a square root $H_{p}$ with eigenvalues of all positive real parts. Consider the state and costate vectors given by

$$
\begin{aligned}
& \mathbf{x}(t)=\left[Q^{-1} W+\exp \left(-H_{p} t\right)\left(I-Q^{-1} W\right)\right] \mathbf{b}, \\
& \mathbf{p}(t)=H_{p} \exp \left(-H_{p} t\right)\left(I-Q^{-1} W\right) \mathbf{b} .
\end{aligned}
$$

It is straightforward to verify that state-costate equation above is satisfied for any initial state $\mathbf{x}(0)=\mathbf{b}$. Moreover, the final condition $\lim _{t \rightarrow \infty} \mathbf{p}(t)=\mathbf{0}$ also holds, due to the fact that $H_{p}$ has all its eigenvalues with positive real parts. It follows that $\mathbf{x}(t)=\mathbf{x}^{\inf }(t)$ satisfies the necessary conditions for the infinite horizon game $\tau \rightarrow \infty$. In order for these to constitute the unique solution for that game, we may revert to counterparts for $n$ players of Theorems 7.16 and 7.10 in [13] and show that the corresponding matrix " $M$ " has a strongly stable solution. The fact that this can be done in a number of special cases suggests that the limiting Nash solution of Theorem 2(iii) is also the unique solution of the infinite horizon game.

\section{References}

1. Acemoglu D, Ozdaglar A (2011) Opinion dynamics and learning in social networks. Dyn Games Appl 1(1):3-49

2. Albi G, Pareschi L, Toscani G, Zanella M (2017) Recent advances in opinion modeling: control and social influence. Act Part 1:49-98

3. Altafini C (2013) Consensus problems on networks with antagonistic interactions. IEEE Trans Autom Control 58(4):935-946

4. Amelkin V, Bullo F, Singh AK (2017) Polar opinion dynamics in social networks. IEEE Trans Autom Control 62(11):5650-5665

5. Axelrod R (1997) The dissemination of culture: a model with local convergence and global polarization. J Conflict Resolut 41(2):203-226

6. Başar T, Olsder GJ (1998) Dynamic noncooperative game theory. SIAM, Philadelphia, PA 
7. Bikhchandani S, Hirshleifer D, Welch I (1992) A theory of fads, fashion, custom, and cultural change as informational cascades. J Polit Econ 100(5):992-1026

8. Bindel D, Kleinberg J, Oren S (2015) How bad is forming your own opinion? Games Econ Behav 92:248-265

9. Blondel VD, Hendrickx JM, Tsitsiklis JN (2010) Continuous-time average-preserving opinion dynamics with opinion-dependent communications. SIAM J Control Optim 48(8):5214-5240

10. Boccaletti S, Bianconi G, Criado R, Del Genio CI, Gómez-Gardenes J, Romance M, Sendina-Nadal I, Wang Z, Zanin M (2014) The structure and dynamics of multilayer networks. Phys Rep 544(1):1-122

11. Deffuant G, Neau D, Amblard F, Weisbuch G (2000) Mixing beliefs among interacting agents. Adv Complex Syst 3:87-98

12. DeGroot MH (1974) Reaching a consensus. J Am Stat Assoc 69(345):118-121

13. Engwerda J (2005) LQ dynamic optimization and differential games. Wiley, West Sussex

14. Etesami SR, Başar T (2015) Game-theoretic analysis of the Hegselmann-Krause model for opinion dynamics in finite dimensions. IEEE Trans Autom Control 60(7):1886-1897

15. Ferraioli D, Goldberg PW, Ventre C (2016) Decentralized dynamics for finite opinion games. Theoret Comput Sci 648:96-115

16. Friedkin NE, Johnsen EC (1990) Social influence and opinions. J Math Sociol 15(3-4):193-206

17. Gabbay M (2007) The effects of nonlinear interactions and network structure in small group opinion dynamics. Physica A 378(1):118-126

18. Ghaderi J, Srikant R (2014) Opinion dynamics in social networks with stubborn agents: Equilibrium and convergence rate. Automatica 50(12):3209-3215

19. Groeber P, Lorenz J, Schweitzer F (2014) Dissonance minimization as a microfoundation of social influence in models of opinion formation. J Math Sociol 38(3):147-174

20. Hegselmann R, Krause U (2002) Opinion dynamics and bounded confidence models, analysis, and simulation. J Art Soc Soc Simulat 5(3)

21. Heider F (1946) Attitudes and cognitive organization 21(1):107-112

22. Horn RA, Johnson CR (1991) Topics in matrix analysis. Cambridge University Press, Cambridge

23. Horn RA, Johnson CR (2002) Matrix analysis. Cambridge University Press, Cambridge

24. Huckfeldt R (2007) Unanimity, discord, and the communication of public opinion. Am J Polit Sci 51(4):978-995

25. Iñiguez G, Török J, Yasseri T, Kaski K, Kertész J (2014) Modeling social dynamics in a collaborative environment. EPJ Data Sci 3:7

26. Jackson MO (2010) Social and economic networks. Princeton University Press, Princeton

27. Kirk DE (1970) Optimal control theory: an introduction. Prentice-Hall, Englewood Cliffs, NJ

28. Krackhardt D (2009) A plunge into networks. Science 326(5949):47-48

29. Lorenz J (2007) Continuous opinion dynamics under bounded confidence: a survey. Int J Mod Phys C 18(12):1819-1838

30. Macy MW, Flache A (2009) Social dynamics from the bottom up: Agent-based models of social interaction. In: The Oxford handbook of analytical sociology. Oxford University Press, pp 245-268

31. Mirtabatabaei A, Bullo F (2012) Opinion dynamics in heterogeneous networks: convergence conjectures and theorems. SIAM J Control Optim 50(5):2763-2785

32. Morarescu IC, Girard A (2011) Opinion dynamics with decaying confidence: application to community detection in graphs. IEEE Trans Autom Control 56(8):1862-1873

33. Niazi MUB (2017) A noncooperative dynamic game model of opinion dynamics in multilayer social networks. Dissertation, Bilkent University, Turkey

34. Niazi MUB,Özgüler AB, Yildiz A (2016) Consensus as a Nash equilibrium of a dynamic game. In: 2016 12th international conference on signal-image technology and internet-based systems (SITIS), IEEE, pp 365-372

35. Olfati-Saber R (2007) Consensus and cooperation in multi-agent networked systems. Proc IEEE 95(1):215-233

36. Osborne MJ, Rubinstein A (1994) A course in game theory. MIT Press, Cambridge

37. Özgüler AB, Yıldız A (2014) Foraging swarms as Nash equilibria of dynamic games. IEEE Trans Cybern 44(6):979-987

38. Parsegov SE, Proskurnikov AV, Tempo R, Friedkin NE (2017) Novel multidimensional models of opinion dynamics in social networks. IEEE Trans Autom Control 62(5):2270-2285

39. Proskurnikov AV, Matveev AS, Cao M (2016) Opinion dynamics in social networks with hostile camps: consensus versus polarization. IEEE Trans Autom Control 61(6):1524-1536

40. Ren W, Cao Y (2011) Distributed coordination of multi-agent networks: emergent problems, models, and issues. Springer, New York 
41. Ren W, Beard RW, Atkins EM (2005) A survey of consensus problems in multi-agent coordination. In: Proceedings of the American control conference, IEEE, pp 1859-1864

42. Shi G, Proutiere A, Johansson M, Baras JS, Johansson KH (2015) Emergent behaviors over signed random dynamical networks: state-flipping model. IEEE Trans Control Netw Syst 2(2):142-153

43. Smith DD (1968) Cognitive consistency and the perception of others' opinions. Public Opin Q 32(1):1-15

44. Yıldız A, Özgüler AB (2015) Partially informed agents can form a swarm in a Nash equilibrium. IEEE Trans Autom Control 60(11):3089-3094

45. Yıldız A, Özgüler AB (2016) Foraging motion of swarms with leaders as Nash equilibria. Automatica 73:163-168

46. Yildiz E, Acemoglu D, Ozdaglar A, Saberi A, Scaglione A (2011) Discrete opinion dynamics with stubborn agents. https://ssrn.com/abstract=1744113

Publisher's Note Springer Nature remains neutral with regard to jurisdictional claims in published maps and institutional affiliations. 